\title{
Joule Heating and Current-Induced Instabilities in Magnetic Nanocontacts
}

\author{
A. Kadigrobov, ${ }^{1,2}$ S. I. Kulinich,,${ }^{1,3}$ R. I. Shekhter,${ }^{1}$ M. Jonson, ${ }^{1}$ and V. Korenivski ${ }^{4}$ \\ ${ }^{1}$ Department of Physics, Göteborg University, SE-412 96 Göteborg, Sweden \\ ${ }^{2}$ Theoretische Physik III, Ruhr-Universität Bochum, D-44780 Bochum, Germany \\ ${ }^{3}$ B. I. Verkin Institute for Low Temperature Physics and Engineering, 47 Lenin Avenue, 61103 Kharkov, Ukraine \\ ${ }^{4}$ Nanostructure Physics, Royal Institute of Technology, SE-10691 Stockholm, Sweden
}

\begin{abstract}
We consider the electrical current through a magnetic point contact in the limit of a strong inelastic scattering of electrons. In this limit local Joule heating of the contact region plays a decisive role in determining the transport properties of the point contact. We show that if an applied constant bias voltage exceeds a critical value, the stationary state of the system is unstable, and that periodic, non-harmonic oscillations in time of both the electrical current through the contact and the local temperature in the contact region develop spontaneously. Our estimations show that the necessary experimental conditions for observing such oscillations with characteristic frequencies in the range $10^{8} \div 10^{9} \mathrm{~Hz}$ can easily be met. We also show a possibility to manipulate upon the magnetization direction of a magnetic grain coupled through a point contact to a bulk ferromagnetic by exciting the above-mentioned thermal-electric oscillations.
\end{abstract}

PACS numbers:

\section{INTRODUCTION: JOULE HEATING AND CURRENT-INDUCED INSTABILITIES IN MAGNETIC NANOCONTACTS.}

Electric transport in mesoscopic magnetic structures has become a hot topic of modern solid state physics research. One reason for this is the prospect for exploiting not only the charge of the electron but also its spin degree of freedom for electronics ("spintronics").

An interplay between macroscopic magnetic degrees of freedom and the electron spin has already been discovered in the giant magnetoresistance (GMR) effect ${ }^{1,2}$. This is a spin-dependent tunnelling phenomenon that quickly found important practical applications in data storage devices. As one shrinks the dimensions of a magnetic material toward the nanometer scale a new, to some extent inverse phenomenon occurs since a high density electric current is able to affect the magnetic order ${ }^{3,4}$. A current-induced precession and switching of magnetization has indeed been observed in a number of experiments on magnetic layers and point contacts $5,6,7,8,9,10$.

Recently ${ }^{11}$ a correlation was observed between the magnitude of a spin-torque effect and electronic scattering in a point contact between a normal and a ferromagnetic metal. Using the method of point contact spectroscopy it was proven that elastic backscattering of electrons plays a crucial role in the transfer of magnetization between differently magnetized regions of the ferromagnetic. This observation also raises the question of the role of inelastic scattering of electrons, including scattering by magnons, in magneto-transport since elastic scattering also shortens the inelastic diffusion length making the transport of heat away from the point contact less effective.

An advantage of using electrical point contacts for switching the direction of magnetization is the possibility to locally achieve extremely high current concentrations of up to $9 \div 10 \mathrm{~A} / \mathrm{cm}^{2}$. Such high current densities are just what is needed for inducing magnetization dynamics. However, there is a restriction due to Joule heating that limits the possibility to increase the current density much further. This is because thermal heating eventually destroys the contact. Nevertheless, for not too high temperatures, a special reversible point-contact transport regime arises in which electric and thermal transport processes leads to a spatial distribution of electric field and temperature that allows the temperature in the point contact to be controlled electrically ${ }^{12,13,14}$. Heating may, however, significantly affect the resistivity of the material creating a nonlinear point-contact transport regime. As we will show below such a nonlinear response to an applied DC voltage can lead to an electrical instability and to time dependent (oscillatory) transport phenomena.

It is well known that strong Joule heating of a metal can result in an $N$ - or $S$-shaped current-voltage $(I-V)$ characteristics containing segments with negative differential conductance (see, e.g., the review 15). In particular, Joule heating of an anti-ferromagnetic break-junction was recently shown to result in an $N$-shaped $I-V$ curve ${ }^{16}$. This phenomenon takes place if the balance between the power released in the heated area and absorbed by the surrounding medium can be satisfied at three different temperatures (two of them stable and the third unstable). As has been shown theoretically and experimentally, such a bi-stability in bulk metals at low temperatures results in spontaneously formed thermo-electric domains $17,18,19,20,21$, nonlinear periodic oscillations 22 and filaments ${ }^{23}$ of high temperature and current in the sample. This effect can be of prime importance for magnetic point contacts and particularly for those made of ferromagnetic manganites. This is because the temperature dependence of their conductance is strong and diverse up to the Curie temperature and above, which may result in $N$-, $S$-shaped $I-V$ characteristics as well as their combinations.

In the present paper we investigate an electrical in- 
stability caused by Joule heating of a magnetic pointcontact junction. We show that non-harmonic periodic oscillations in time of the total current and the voltage drop across the Joule-heated part of the micro-contact (as well as the local temperature at the contact) appear spontaneously if the voltage bias of the entire sample is kept constant. This phenomenon takes place in a wide interval of sample temperatures that includes the Curie temperature of the ferromagnetic conductor. We also show that in the latter case the magnetization direction of a magnetic grain (which is coupled to a bulk ferromagnetic through a point-contact) periodically switches from the direction of the bulk ferromagnetic to the direction of the external magnetic field following periodic heating and cooling of the point-contact under the regime of the thermal-electric self-exciting oscillations.

\section{FORMULATION OF THE PROBLEM}

Below we consider the case when the electron relaxation lengths for both momentum and energy are shorter than the size of the micro-contact (shown in Fig. 1). Under this condition the thermal and electrical characteristics obey the continuity equations for the energy

$$
C_{V} \frac{\partial T}{\partial t}+\operatorname{div}(-\kappa(T) \nabla T)=-\sigma(T)(\nabla \varphi)^{2}
$$

and the local charge neutrality condition

$$
\operatorname{div} \mathbf{j}=0,
$$

while the total current $I$ flowing through the system satisfies the equation

$$
\mathcal{L} d I / d t+R I=V
$$

(see, e.g., Ref. 24). Here $C_{V}$ is the heat capacity of the metal per unit volume, $T$ is the temperature, $\kappa$ is the thermal conductivity, $\sigma$ is the electrical conductivity, $\varphi$ is the electric potential, $\mathbf{j}=-\sigma(T) \nabla \varphi$ is the electrical current density, $\mathcal{L}$ is the total inductance of the circuit, $R$ is the total resistance (we assume below that the main contribution to $R$ is from the point-contact) and $V$ is the applied bias voltage. In writing Eq. (10) we neglected the thermopower, which is small by a factor $k_{\mathrm{B}} T / \epsilon_{F}$ where $k_{\mathrm{B}}$ is the Boltzmann constant and $\epsilon_{\mathrm{F}}$ is the Fermi energy.

The boundary conditions for the set of equations (1) and (2) are determined by the absence of heat and charge transport through the boundaries of the contact. It follows that

$$
j_{n}(\mathbf{r} \in \Sigma) \equiv \mathbf{n} \cdot \mathbf{j}=0, \quad j_{\mathrm{q}, n}(\mathbf{r} \in \Sigma) \equiv \mathbf{n} \cdot \mathbf{j}_{q}=0,
$$

where $\mathbf{j}_{\mathrm{q}}=-\kappa \nabla T+\varphi \mathbf{j}$ and $\mathbf{n}$ is the normal to the boundary $\Sigma$ of the micro-contact. For a symmetric contact one has

$$
\varphi(z \rightarrow \pm \infty)= \pm \frac{V}{2} ; \quad T(z \rightarrow \pm \infty)=T_{0} .
$$

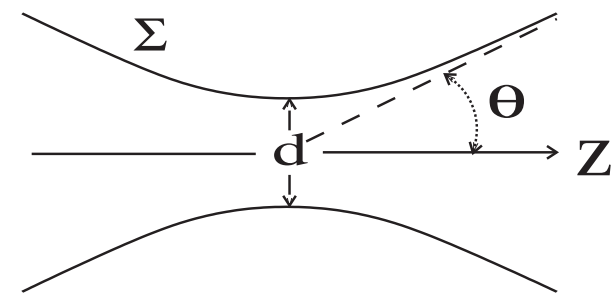

FIG. 1: Model of a micro-contact whose transport properties are discussed in the text.

Here $T_{0}$ is the temperature of the peripheral regions of the contact, the $z$-axis is directed along the axis of the contact.

As was shown in Ref. 25 it is mathematically convenient to use the coordinates $u, v, \phi$ of an oblate ellipsoid for studying the kinetic properties of micro-contacts. These coordinates are related to the Cartesian coordinates $x, y, z$ as

$$
\begin{aligned}
& x=d_{0} \sin u \cosh v \cos \phi, y=d_{0} \sin u \cosh v \sin \phi \\
& z=d_{0} \cos u \sinh v
\end{aligned}
$$

where $0 \leq u \leq \theta,-\infty<v<\infty, 0 \leq \phi \leq 2 \pi, u=$ $\theta=$ const at the boundary $\Sigma$ of the micro-contact, $d_{0}=$ $d /(2 \sin \theta)$ is the effective length of the contact and $d$ is the smallest diameter of the contact as indicated in Fig. 1]

We assume the contact to be symmetric and hence the temperature and the electric potential depend only on the coordinate $v$ and time $t$. Therefore, Eqs. (1) and (2), expressed in the new coordinates, reduce to

$$
\begin{gathered}
C_{V} \frac{\partial T}{\partial t}-\frac{1}{d_{0}^{2} \sinh ^{2} v \cosh v} \frac{\partial}{\partial v}\left[\kappa(T) \cosh v \frac{\partial T}{\partial v}\right] \\
-\frac{\sigma(T)}{d_{0}^{2} \sinh ^{2} v}\left(\frac{\partial \varphi}{\partial v}\right)^{2}=0 \\
\frac{\partial}{\partial v}\left[\sigma(T) \cosh v \frac{\partial \varphi}{\partial v}\right]=0
\end{gathered}
$$

While deriving Eq. (8) we neglected the term $\cos ^{2} u$ in the sum $\sinh ^{2} v+\cos ^{2} u$ (which arises in the Lame coefficients). This is a reasonable approximation if the characteristic size of the region of Joule heating is large enough.

Integrating Eqs. (7), (8) and the relation $\mathbf{j}=-\sigma(T) \nabla \varphi$ over the cross-section area of the sample one gets a set of equations that describe the space- and time-evolution of the temperature $T(\eta, t)$ and the total current $I(t)$ of a Joule-heated microcontact,

$$
f(\eta) C_{V} \frac{\partial T}{\partial t}-\frac{\partial}{\partial \eta}\left[\kappa(T) \frac{\partial T}{\partial \eta}\right]-\rho(T) I^{2} / l_{0}^{2}=0 ;
$$

$$
\mathcal{L} \frac{d I}{d t}+R I=V ; \quad R=\langle\rho(T)\rangle
$$


In Eq. (9), where $f(\eta)=d_{0}^{2} \sinh ^{2} 2 v(\eta) / 4$ and $l_{0}^{2}=$ $2 \pi d_{0}^{2}(1-\cos \theta)$, we introduced a new variable $\eta$ that is related to $v$ through the relation

$$
\frac{\partial}{\partial \eta}=\cosh v \frac{\partial}{\partial v}
$$

while in Eq. (10) and below, angular brackets imply an integration over $\eta$,

$$
\langle\ldots\rangle=\frac{1}{l_{0}} \int_{-\pi / 2}^{-\pi / 2} \ldots d \eta
$$

\section{NEGATIVE DIFFERENTIAL RESISTANCE AND STABILITY OF THE STATIONARY TEMPERATURE AND CURRENT IN A JOULE-HEATED MICROCONTACT.}

The set of equations (9) and (10) subject to the boundary conditions (5) always have steady-state solutions $I=\bar{I}(V)$ and $T=\bar{T}(V, \eta)$ that satisfy the stationary equations

$$
\begin{gathered}
-\frac{\partial}{\partial \eta}\left[\kappa(\bar{T}) \frac{\partial \bar{T}}{\partial \eta}\right]-\rho(\bar{T}) \bar{I}^{2} / l_{0}^{2}=0 \\
V=R \bar{I}
\end{gathered}
$$

According to Refs. 26 and 27 the stationary currentvoltage characteristics of the microcontact is

$\bar{I}(V)=\frac{V}{R(V)}, \quad R^{-1}(V)=\frac{l_{0}}{\pi} \int_{0}^{1} d x / \rho\left(T_{\mathrm{m}}(V) \sqrt{1-x^{2}}\right)$

where $T_{\mathrm{m}}$ is the temperature in the center of the microcontact $(\eta=0)$ and depends on the applied voltage $\operatorname{as}^{26,28}$

$$
T_{\mathrm{m}}^{2}=T_{\mathrm{bulk}}^{2}+\frac{V^{2}}{4 L}
$$

where $L=\pi^{2} k_{B}^{2} /\left(3 e^{2}\right)$ is the Lorentz number. The dependence of the stationary temperature $\bar{T}(V, \eta)$ on the bias voltage $V$ and the coordinate $\eta$ is determined by the relation

$$
R(V) \int_{0}^{\Delta(\bar{T})} d x / \rho\left(T_{\mathrm{m}}(V) \sqrt{1-x^{2}}\right)=\frac{2 \eta}{\pi}
$$

where $\Delta(T)=\sqrt{1-\left(4 L / V^{2}\right)\left(T^{2}-T_{\text {bulk }}^{2}\right)}$

Differentiating Eq. (14) with respect to $V$ one sees that the differential conductance $d I / d V$ is negative if $-R(V)\left(V d R^{-1} / d V\right)>1$. Assuming the resistivity to be $\rho(T)=\rho_{0}+\rho_{1}(T)$, where $\rho_{0}$ is the residual resistivity and the temperature-dependent part $\rho_{1} \propto T^{\alpha}$, one finds the differential conductance to be negative if

$$
\alpha\left[1-R \int\left(\frac{\rho_{0}}{\rho\left(T_{\mathrm{m}} \sqrt{1-x^{2}}\right)}\right) \frac{d x}{\rho\left(T_{\mathrm{m}} \sqrt{1-x^{2}}\right)}\right]>1
$$

If the microcontact is heated to temperatures for which $\rho_{1}\left(T_{\mathrm{m}}\right) \gg \rho_{0}$ the inequality (17) reduces to

$$
\alpha>1+k \frac{\rho_{0}}{\rho\left(T_{\mathrm{m}}\right)},
$$

where $k$ is a constant of order one $(k \sim 1)$. For many ferromagnetic metals $\alpha \approx 2$ and increases as the temperature approaches the Curie temperature (see, e.g., Ref.29) and hence the $I-V$ characteristics of a Joule-heated ferromagnetic microcontact has sections with a negative differential resistivity in a wide range of temperatures including the Curie temperatures.

As soon as the micro-contact is heated to temperatures where the differential resistance is negative, the stationary distribution of temperature and current in the contact may be unstable.

In order to investigate the stability of the stationary temperature $\bar{T}$ and current $\bar{I}$ given by Eqs. (16) and (14) we write the temperature $T(t, \eta)$ and the current $I(t)$ as sums of two terms,

$$
T(t, \eta)=\bar{T}(\eta)+T_{1}(t, \eta), \quad I(t)=\bar{I}+I_{1}(t),
$$

where $T_{1}$ and $I_{1}$ are small corrections to the stationary values. After substituting Eq. (19) into Eqs. (9) and (10) we obtain a linearized set of equations for $\Theta_{1}=\kappa(\bar{T}) T_{1}$ and $I_{1}$ of the form

$$
\begin{aligned}
& \frac{\partial \Theta_{1}}{\partial t}+\beta(\eta) \hat{H} \Theta_{1}=\frac{2 \beta(\eta) \rho(\bar{T}) \bar{I}}{l_{0}} I_{1} ; \\
& \mathcal{L} \frac{\partial I_{1}}{\partial t}+R I_{1}+\delta R \bar{I}=0 ; \quad \delta R=\left\langle\frac{\rho^{\prime}(\bar{T})}{\kappa(\bar{T})} \Theta_{1}\right\rangle,
\end{aligned}
$$

where $\beta=1 /\left(f(\eta) C_{V}(\bar{T})\right)$ and the Hermitian operator is

$$
\hat{H}=-\frac{\partial^{2}}{\partial \eta^{2}}-\frac{\bar{I}^{2} \rho^{\prime}(\bar{T})}{l_{0}^{2} \kappa(\bar{T})}
$$

(here and subsequently, a prime denotes differentiation with respect to $T$ ).

As shown in Appendix A the Laplace transformed current

$$
i_{1}(p)=\int_{0}^{\infty} I(t) \exp \{-p t\} d t
$$

can be expressed as

$$
i_{1}(p)=\left\{I_{1}(0)-\frac{1}{\mathcal{L}} \bar{I} \sum_{\nu} \frac{A_{\nu}(0)}{p+\lambda \nu}\right\} / D(p),
$$

where the denominator is

$$
D(p)=p\left(1-\frac{1}{\mathcal{L}} \frac{2 \bar{I}^{2}}{l_{0}^{2}} \sum_{\nu} \frac{\rho_{\nu} R_{\nu}}{\lambda_{\nu}\left(p+\lambda_{\nu}\right)}\right)+\frac{1}{\mathcal{L}} \frac{d \bar{V}}{d \bar{I}}
$$


Here $A_{\nu}$ are coefficients of the series expansion of $\Theta_{1}$ in eigenfunctions $\psi_{\nu}$ of the Hermitian operator $\hat{H}_{\beta}=$ $\beta(\nu) \hat{H}$, and $\lambda_{\nu}$ are the eigenvalues of this operator (see Eq. (A2)).

It follows from Eqs. (23) and (24) that for the stationary current $\bar{I}$ and temperature $\bar{T}$ (see Eqs. (14) and (16)) to be stable it is necessary and sufficient that the function $D(p)$ has no zeros in the half-plane $\operatorname{Re} p>0$. It is easy to see that the stationary current and temperature are stable if the inductance $\mathcal{L}$ is small, that is $\tau_{I} \ll \tau_{T}$ where

$$
\tau_{I}=\frac{\mathcal{L}}{R} ; \quad \tau_{T}=\frac{d_{0}^{4}}{\bar{I}^{2}} \frac{\bar{T} C_{V}(\bar{T})}{\rho(\bar{T})}
$$

are the characteristic relaxation times for current and temperature, respectively. In this case $D(p)=0$ at $p \propto$ $1 / \tau_{I}$ and hence one can expand $D(p)$ as a power series in $p^{-1}$ and find $I_{1}(t) \propto \exp \left(p_{1,2} t\right)$ where

$$
p_{1,2}=\frac{1}{2 \mathcal{L}}\left(-R \pm \sqrt{R^{2}-\frac{8 \bar{I}}{l_{0}} \sum_{\nu} \rho_{\nu} \tilde{R}_{\nu}}\right)
$$

that is the stationary distribution is stable at low inductances.

In the opposite case of large $\mathcal{L}\left(\tau_{I} \gg \tau_{T}\right)$ one can neglect the second term in Eq. (24) and find

$$
I_{1}(t) \propto \exp \left\{-\frac{1}{\mathcal{L}} \frac{d \bar{V}}{d \bar{I}} t\right\}
$$

From Eq. (27) it follows that the stationary current and the temperature are always stable in the branches of the $I-V$ curve that have a positive differential resistance, and they are unstable in the branches with a negative differential resistance. In the next section we investigate the adiabatic evolution of this instability in the case of a large inductance $\mathcal{L}$.

\section{THERMO-ELECTRIC SELF-EXCITED PERIODIC OSCILLATIONS OF THE TOTAL CURRENT AND OF THE VOLTAGE DROP OVER THE JOULE-HEATED PART OF THE MICROCONTACT}

As was shown in Section III the stationary current $\bar{I}$ and temperature $\bar{T}(\eta)$ in a microcontact (Eq. (14) and Eq. (16)) become unstable if $\tau_{I}>\tau_{T}$. Below we show that this instability results in the spontaneous appearance of non-harmonic periodic oscillations in time of the total current $I$ flowing through the microcontact and of the voltage drop $U$ over the Joule-heated part of the contact provided the applied bias voltage $V$ is kept fixed. In Subsection IVA we derive a set of reduced adiabatic equations for $I$ and $U$; in Subsection IVB we study solutions of these equations both analytically and numerically.

\section{A. Adiabatic evolution equations}

In order to investigate the evolution of the instability (which is governed by Eqs. (9) and (10) we assume that the inductance $\mathcal{L}$ is large enough to have $\tau_{I} \gg \tau_{T}$. In this case the current varies slowly in time while the temperature rapidly follows the current varations. This assumption allows us to develop an adiabatic perturbation theory in which the temperature and the current can be represented as

$$
\begin{aligned}
T(\eta, t) & =\tilde{T}(U(t), \eta)+\tilde{T}_{1}(\eta, t) \\
I(t) & =\tilde{I}(t)+\tilde{I}_{1}(t) .
\end{aligned}
$$

Here $\tilde{T}(U(t), \eta)$ is the steady-state solution (16) of Eqs. (12) and (13) in which the constant voltage $V$ has been replaced by a time-dependent voltage $U(t)$ (to be determined). Hence $\tilde{T}(U(t), \eta)$ satisfies the equation

$$
\begin{array}{r}
-\frac{\partial}{\partial \eta}\left[\kappa(\tilde{T}) \frac{\partial \tilde{T}}{\partial \eta}\right]-\rho(\tilde{T}) \bar{I}^{2}(U) / l_{0}^{2}=0 \\
\bar{I}(U)=U / R(U)
\end{array}
$$

where

$$
R(U)=R\{\tilde{T}\}=\langle\rho(\tilde{T})\rangle
$$

while the current $\tilde{I}(t)$ satisfies the equation

$$
\mathcal{L} \frac{\partial \tilde{I}}{\partial t}+R\{\tilde{T}\} \tilde{I}=V
$$

An equation for $U(t)$ can be found from the condition that $\tilde{T}_{1}$ and $\tilde{I}_{1}$ are small corrections to $\tilde{T}$ and $\tilde{I}$, respectively (that is $\left|\tilde{T}_{1}\right| \ll \tilde{T}$ and $\left|\tilde{I}_{1}\right| \ll \tilde{I}$ ). As shown in Appendix $\mathrm{B}$ this condition is satisfied if the current $\tilde{I}(t)$ and the voltage $U(t)$ satisfy the following set of ordinary differential equations:

$$
\begin{aligned}
& \mu \frac{d U}{d t}=\tilde{I}^{2}-\bar{I}^{2}(U) \\
& \mathcal{L} \frac{d \tilde{I}}{d t}+R(U) \tilde{I}=V
\end{aligned}
$$

Here

$$
\mu=4\left(\frac{d R}{d U}\right)^{-1}\left(\frac{d \bar{I}(U)}{d U} \frac{\bar{I}}{l_{0}}\right)^{2} \sum_{\nu} \frac{\tilde{R}_{\nu} \tilde{\rho}_{\nu}}{\tilde{\lambda}_{\nu}^{2}} \sim \frac{V}{R^{2}} \tau_{T}
$$

and $V$ is the applied bias voltage; the $\bar{I}-U$ curve $\bar{I}(U)=U / R(U)$ and the resistance $R(U)$ are determined explicitly by Eq. (14) and Eq. (15) in which $V$ has to be changed to $U(t)$.

The set of non-linear ordinary differential equations Eq.(32) allows the system under consideration to be mapped onto the effective circuit shown in Fig.(2). The circuit includes a Joule-heated conductor in series with 


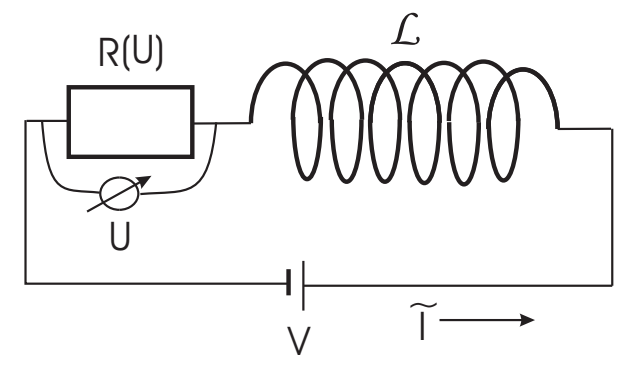

FIG. 2: An effective circuit under a fixed voltage $V$ containing a Joule-heated conductor in series with an inductance $\mathcal{L}$; the resistance $R(U)$ depends on the voltage drop $U$ on the conductor in such a way that the conductor CVC $\bar{I}(U)=U / R(U)$ has a section with a negative differential resistance; $\tilde{I}$ is the total current flowing in the circuit.

an inductance $\mathcal{L}$, the total voltage drop in the circuit $V$ being kept constant in time. The Joule-heating of the conductor is assumed to be inhomogeneous, so that the relationship between its temperature and voltage $U$ is described by the thermal continuity equation Eq. (28) and hence the rate of heat removal from the conductor is $Q=R \bar{I}^{2}(U)$.

In the next Subsection we investigate the conditions under which the steady-state solution of Eq. (32) is unstable and how it evolves into a limit cycle in the $\tilde{I}-U$ plane, a limit cycle that corresponds to periodic nonharmonic oscillations of the current and the voltage drop over the Joule-heated part of the micro-contact.

\section{B. Spontaneous development of periodic} oscillations of the current and of the voltage drop over the point contact

Let the steady-state solution of the set of equations (32) be

$$
\tilde{I}_{0}=\bar{I}\left(U_{0}\right) ; \quad U_{0}=V
$$

A study of the stability of the steady-state solution (34) with respect to small perturbations, carried out on the basis of the linearized set of equations (32) shows that small variations $I_{1}$ and $U_{1}$ from the steady-state develop as $\exp \left(\gamma_{1,2} t\right)$ with time, where the rate factor

$$
\gamma_{1,2}=-\left(\frac{1}{\mathcal{L}} R+\frac{d \bar{I}}{d V} \frac{\bar{I}}{\mu}\right) \pm \sqrt{\left(\frac{1}{\mathcal{L}} R+\frac{d \bar{I}}{d V} \frac{\bar{I}}{\mu}\right)^{2}-\frac{2 \bar{I}}{\mu \mathcal{L}}}
$$

From Eq. (35) one sees that in the case of a negative differential conductance the steady-state solution looses its stability with an increase of the inductance as soon as the term in the round brackets changes sign, i.e. as soon as $\mathcal{L}>\mathcal{L}_{\text {cr }}$, where the critical value of the inductance is

$$
\mathcal{L}_{\mathrm{cr}}=\mu \frac{R}{|\bar{I}|}\left|\frac{d V}{d \bar{I}}\right|
$$

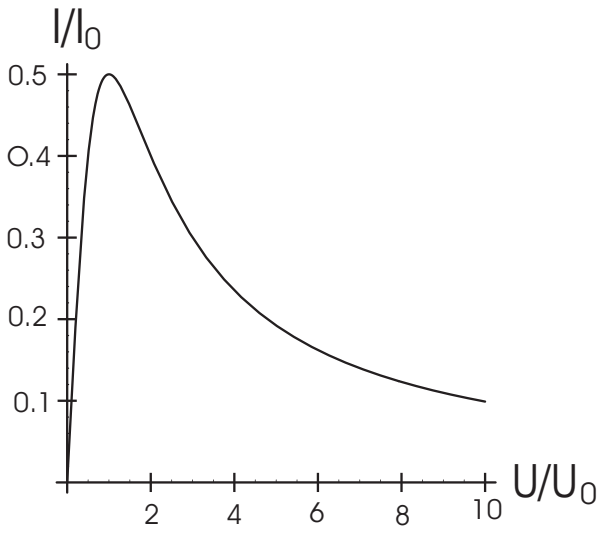

FIG. 3: Dependence of the total current $I$ through the microcontact shown in Fig. 1 on the voltage drop $U$ over the contact for the case that the micro-contact resistance depends on voltage as $R(U)=\left(R_{0}+\beta U^{2}\right)$. The current is normalized to $I_{0}=U_{0} / R_{0}$, where $U_{0}=\sqrt{R_{0} / \beta}$

Comparing Eq. (36) with Eq. (25) one sees that stability is lost as soon as $\tau_{I}>\tau_{T}$ in accordance with the above analysis. Therefore, if $\tau_{I}>\tau_{T}$ any initial state close to the steady state $U_{0}, I_{0}$ is repelled from this point in the voltage-current plane. On the other hand, as one easily sees from Eq. 32 any initial state which is very far from the stable point (that is $|I(t=0)| \gg\left|I_{0}\right|$ or $\left.|U(t=0)| \gg\left|U_{0}\right|\right)$ decreases in time and is attracted to the stable point. It means that there is a stable limit circle in the $I-U$ plane (see, e.g., Ref. 30). This means that non-linear periodic oscillations of the total current and the voltage drop over the micro-contact appear spontaneously if the bias voltage $V$ is kept fixed. In order to show characteristic features of the spontaneous development of electric oscillations, we present a typical currentvoltage characteristics for a micro-contact in Fig. 3 and the corresponding spontaneous oscillation cycle of the current through the system and the voltage drop over the Joule-heated micro-contact is shown in Fig 4

Simple estimations of the ratio $\tau_{I} / \tau_{T}$ (see Eq. 250) show that the critical value of the inductance $\mathcal{L}_{c r}$ (at which $\tau_{I} / \tau_{T} \sim 1$ ) is

$$
\mathcal{L}_{c r} \sim \frac{T C_{V}}{j_{0}^{2} d_{0}}
$$

where $j_{0}$ is the characteristic value of the current density in the micro-contact and $d_{0}$ is the size of the microcontact.

\section{Magnetization switching under the thermal-electric self-exciting oscillations.}

The system under consideration is presented in Fig 5 A conducting magnetic grain is coupled through a point- 


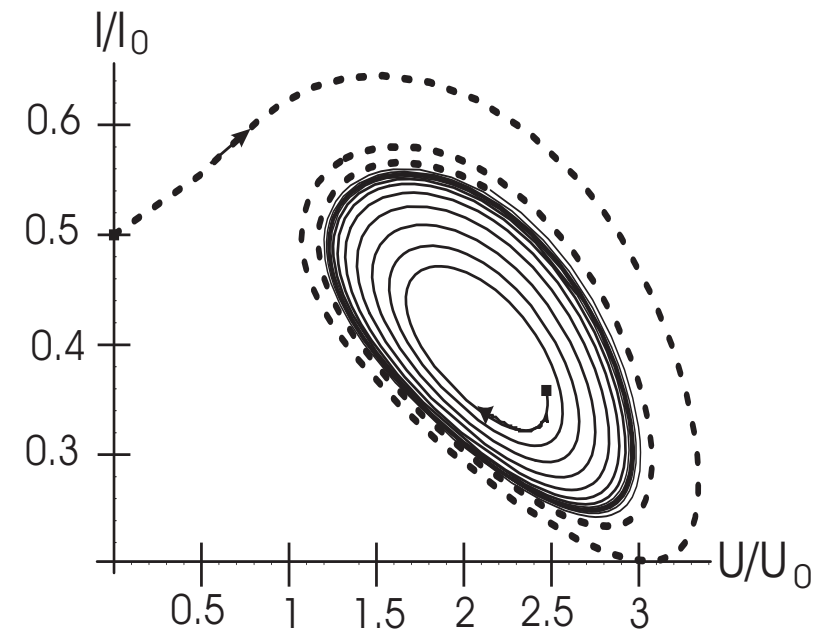

FIG. 4: Spontaneous oscillations of the current $I(t)$ trough the micro-contact sketched in Fig. 1 and the voltage drop $U(t)$ over the contact. Here $I_{0}=U_{0} / R_{0}$ and $U_{0}=\sqrt{R_{0} / \beta}$. Initial values of $I(0)$ and $U(0)$ are shown by filled squares. As time goes on the current and the voltage drop move along the dashed or thin solid line towards the limit cycle (thick solid line) depending on whether the initial state is outside or inside the limit cycle. After a time $t \gg \tau_{I}$ the set $(I(t), U(t))$ moves along the limit cycle executing a periodic motion, i.e. the current $I(t)$ and the voltage drop $U(t)$ execute periodic non-linear oscillations.

contact (PC) to a bulk magnetic conductor and to a nonmagnetic conductor (the right-hand side of the figure). There is a magnetic field $H$ directed opposite to the magnetization of the bulk magnetic conductor. The magnetic field is weak enough so that at low temperatures the grain magnetization is parallel to the magnetization of the bulk ferromagnetic due to the exchange interaction.

Let the inductance of the circuit to be large enough in order that the above-mentioned thermal-electric selfoscillations arise in the system. The limiting cycle along which the current $J$ and the voltage drop $U$ periodically move in time are shown in Fig [6] (for the sake of simplicity it is shown for the case of a extremely large inductance $\left.\tau_{\mathcal{L}} \gg \tau_{T}\right)$.

When $J$ and $U$ move along the left arising branch of the CVC the PC is cold (its temperature is nearly the same as those of the cooling media). When the system jumps to the right arising branch of the CVC (along the upper straight line in Fig 6), the PC is Joule heated above the Curie temperature while the bulk ferromagnetic and the grain remain cold. It means that the magnetization in the vicinity of the PC disappears and hence the exchange coupling of the grain to the bulk ferromagnetic is interrupted, and the magnetization of the grain flips to the direction of the magnetic field $H$. Moving further along the limiting cycle (along the right branch of CVC), the system jumps to the left arising branch of the CVC
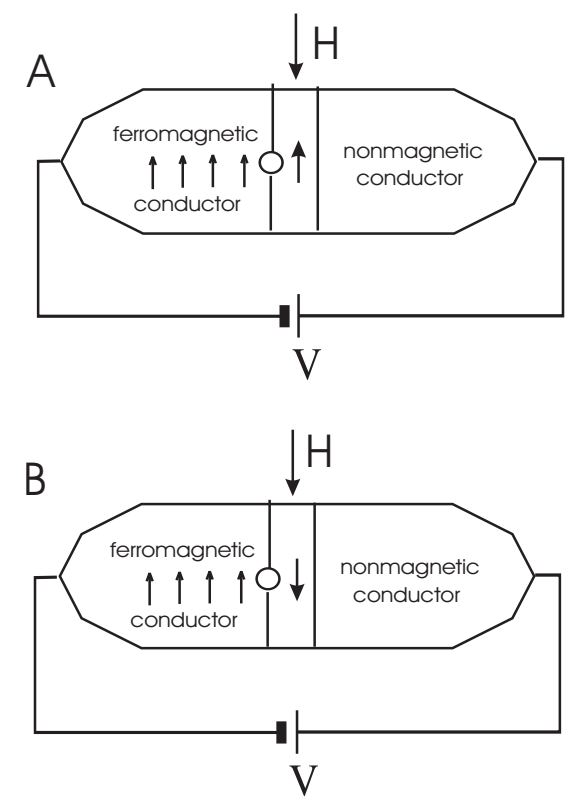

FIG. 5: A.The nanocontact is cold. The exchange coupling through the contact keeps the magnetization of the grain parallel to the magnetization of the left bulk ferromagnetic conductor. B The nanocontact is hot. The exchange coupling through the nanocontact is killed and the magnetic field $H$ flips the magnetization of the grain.

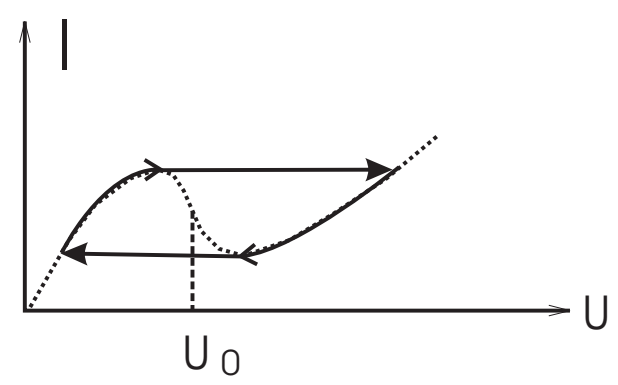

FIG. 6: The limiting cycle for an extremely large inductance in the circuit $\left(\tau_{\mathcal{L}} \gg \tau_{T}\right)$; when the total current $I(t)$ and the voltage drop $U(t)$ on the point-contact move along the left branch of $\mathrm{CVC}$ the point contact is cooled to temperatures close to the thermostat temperature; while they move along the right branch of CVC the point contact is heated above the Curie temperature.

(along the lower straight line in Fig 60), the PC is cooled to temperatures close to the thermostat temperature, the magnetization in the vicinity of the PC is restored, and the grain magnetization flips to the direction of the bulk magnetization again. After that these flips repeat periodically in time with the periodicity of the thermal-electric self-exciting oscillations.

The period of the thermal-electric self-oscillations and hence the period of the switching of the grain magnetization is controlled by the inductance in the circuit (as well as by the applied voltage). 
The above-mentioned mechanism of the magnetization switching works if, at low temperatures, the exchange coupling of the grain to the bulk ferromagnetic keeps the grain magnetization parallel to the bulk magnetization despite the opposite direction of the applied magnetic field. By this is meant that if at low temperatures, the grain magnetization flips to the direction of the magnetic field the total energy of the system increases, that is

$$
W_{\text {total }}=\int d V\left(\alpha\left(\frac{\partial \vec{M}}{\partial x_{i}}\right)^{2}-\vec{M} \vec{H}\right)>0
$$

Here $\vec{M}$ is the magnetic moment, $\alpha \sim I / a M^{2}$ where $I \sim k T_{c}$ and $a$ are the exchange energy and the lattice spacing, respectively, $k$ is the Boltzmann constant and $T_{c}$ is the Curie temperature. If the characteristic size of the $\mathrm{PC} d$ is less than the domain wall length $l_{d w}$, the length of the effective domain wall is of the order of $d$ ( $\left.\sec ^{31}\right)$, and from Eq.(38) it follows that $W_{\text {total }}>0$ if $\alpha\left(M^{2} / d^{2}\right) d^{3}-\left(\hbar \omega_{H} / a^{3}\right) V_{0}>0$ that is

$$
V_{0}<\frac{T_{c}}{T_{H}}\left(a^{2} d\right)
$$

where $V_{0}$ is the volume of the grain, $T_{H}=\hbar \omega_{H} / k$ and $\omega_{H}=e H /(m c)$ ( $e$ is the electron charge and $m$ is the electron mass). If one takes, e.g., $d \sim 10 a, T_{c} \sim 10^{3} \mathrm{~K}$, $H \sim 10^{2} \div 10^{3}$ gauss (that is $10^{-3} \div 10^{-2}$ T)and $V_{0} \sim$ $10 a \times L^{2}$ one finds the characteristic width of the grain $L \sim\left(10^{2} \div 10^{3}\right) a$ where $a$ is the atomic spacing in the crystal.

\section{CONCLUSION}

In conclusion we have theoretically studied the temperature dependent electrical current through a voltagebiased point contact. We predict that spontaneous temporal oscillations of the temperature and current through the contact may appear due to Joule heating and a strong temperature dependence of the point contact resistance.

We have also showed that the self-exciting oscillations can control the magnetization direction of a magnetic grain coupled through a point-contact to a bulk magnetic conductor: the grain magnetization direction periodically switches from the direction of the bulk magnetization to the direction of the external magnetic field following periodic cooling and heating of the point contact under the regime of the self-exciting thermal-electric oscillations. In order to estimate the characteristic frequency of these self-oscillations we take the minimum width $d$ of the micro-contact shown in Fig. 1 to be $200 \mathrm{~nm}$ and let the current $I$ through the contact be $161 \mathrm{~mA}$, its temperature $T \sim 30 \mathrm{~K}$ and the specific heat $C_{V}$ at this temperature ${ }^{20} \sim 1 \mathrm{Jcm}^{-3} \mathrm{~K}^{-1}$. Under these conditions we can use Eq. 36] to evaluate the critical inductance $\mathcal{L}_{\mathrm{cr}}$ required for self-oscillations to develop as well as the oscillation frequency $\omega$ when $\mathcal{L} \gtrsim \mathcal{L}_{c r}$. The result is

$$
\mathcal{L}_{c r} \sim 10^{-6} \div 10^{-5} H ; \quad \omega \sim 10^{8} \div 10^{9} s^{-1}
$$

and we note that the frequency of the self-oscillations decreases linearly with an increase of the inductance of the circuit.

Finally we observe that due to Joule heating of the contact area, where the current density is maximal, a wide range of temperatures can be reached in this region of the sample (and controlled by the bias voltage). This allows for a thermal scanning that avoids difficult calorimetric measurements in many cases and since the Joule heating easily can produce temperatures of the order of the critical temperature for magnetic phase transitions, it offers an exciting possibility for electrically controlled magnetic switching on the nanometer scale.

Acknowledgment - Financial support from the Swedish KVA, VR and SSF is gratefully acknowledged.

\section{APPENDIX A}

Let us write $\Theta_{1}(\eta, t)$ in the form of an expansion as

$$
\Theta_{1}(\eta, t)=\sum_{\nu} A_{\nu}(t) \psi_{\nu}(\eta)
$$

where $\psi_{\nu}$ are the eigenfunctions of the operator $\hat{H}_{\beta}=$ $\beta(\eta) \hat{H}$, which satisfies the Sturm-Liouville equation

$$
\left[-\frac{\partial^{2}}{\partial \eta^{2}}-\frac{\bar{I}^{2} \rho^{\prime}(\bar{T})}{l_{0}^{2} \kappa(\bar{T})}\right] \psi_{\nu}=\lambda_{\nu} \beta^{-1} \psi_{\nu}
$$

Substituting Eq. (A1) into Eqs. (20)-(B3) and carrying out a Laplace transformation with respect to $t$ we find the following set of algebraic equations for $a(p)=$ $\int_{0}^{\infty} A(t) \exp (-p t) d t$ and $i_{1}(p)=\int_{0}^{\infty} I(t) \exp (-p t) d t$ :

$$
\begin{aligned}
\left(p+\lambda_{\nu}\right) a_{\nu}-\left(2 \bar{I} / l_{0}^{2}\right) \rho_{\nu} i_{1} & =A_{\nu}(0) \\
(\mathcal{L} p+R) i_{1}+\bar{I} \sum_{\nu} R_{\nu} a_{\nu} & =\mathcal{L} I_{1}(0) .
\end{aligned}
$$

Here $A_{\nu}(0)$ and $I_{1}(0)$ are the initial values of $A_{\nu}$ and $I_{1}$ at $t=0$, respectively, while

$$
R_{\nu}=\left\langle\frac{\rho^{\prime}(\bar{T})}{\kappa(\bar{T})} \psi_{\nu}(\eta)\right\rangle ; \quad \rho_{\nu}=\left\langle\rho(\bar{T}) \psi_{\nu}(\eta)\right\rangle
$$

While obtaining Eq. A3 we took into account the fact that the Hermitian conjugate operator $\hat{H}_{\beta}^{\dagger}=\hat{H} \beta(\eta)$ has eigenfunctions $\bar{\psi}_{\nu}=\beta^{-1} \psi_{\nu}$ and hence $\left\langle\psi_{\nu}(1 \beta) \psi_{\nu^{\prime}}\right\rangle=$ $\delta_{\nu, \nu^{\prime}}$.

On the other hand, differentiating the current-voltage characteristics $V=\langle\rho(\bar{T}(\bar{I}, \eta)\rangle \bar{I}$ and Eq. (12) with respect to the current $\bar{I}$ one obtains

$$
\frac{d V}{d \bar{I}}=R+\bar{I}\left\langle\rho \frac{\partial \bar{T}}{\partial \bar{I}}\right\rangle
$$

and

$$
\hat{H}\left(\kappa(\bar{T}) \frac{\partial \bar{T}}{\partial \bar{I}}\right)=\frac{2 \bar{I}}{l_{0}^{2}} \rho
$$


Here $\hat{H}$ is the operator defined by Eq. (22). It follows from Eq. A51 and Eq. (A2) that $\kappa d \bar{T} / d \bar{I}$ can be written in the form

$$
\kappa \frac{\partial \bar{T}}{\partial \bar{I}}=\frac{2 \bar{I}}{l_{0}^{2}} \sum_{\nu} \frac{\rho_{\nu}}{\lambda_{\nu}} \psi_{\nu}
$$

Substituting Eq. A6 into Eq. (A4) we find

$$
\frac{d \bar{V}}{d \bar{I}}=R+\frac{2 \bar{I}}{l_{0}^{2}} \sum_{\nu} \frac{\rho_{\nu} R_{\nu}}{\lambda_{\nu}}
$$

Using Eq. A3 and the equality Eq. A7 one gets Eq. (223)

\section{APPENDIX B}

Inserting first Eq. (28) into Eqs. (9) and (10) we obtain linear differential equations for $\tilde{T}_{1}$ and $\tilde{I}_{1}$ of the form

$$
\begin{aligned}
\hat{H}_{\beta}\left(\kappa \tilde{T}_{1}\right)-2 \tilde{\beta} \rho(\bar{T}) \frac{\bar{I}(U)}{l_{0}^{2}} \tilde{I}_{1} & =\tilde{\beta} F \\
\mathcal{L} \frac{d \tilde{I}_{1}}{d t}+R\{\tilde{T}\} \tilde{I}_{1}+\delta \tilde{R} \tilde{I} & =0
\end{aligned}
$$

where

$$
\begin{aligned}
F & \equiv \frac{\rho(\tilde{T})}{l_{0}^{2}}\left(\tilde{I}^{2}-\bar{I}^{2}(U)\right)-\frac{1}{\tilde{\beta}} \frac{d \tilde{T}}{d U} \frac{d U}{d t}, \\
\delta \tilde{R} & =\left\langle\rho^{\prime}(\tilde{T}) \tilde{T}_{1}\right\rangle ; \quad \tilde{\beta}=\beta(\tilde{T}) .
\end{aligned}
$$

When deriving the above equations we neglected a term $\propto d T_{1} / d t$ in Eq. (B1) since it is of second order in the parameter $\gamma=\tau_{T} / \tau_{I} \ll 1$ (in contrast to $\mathcal{L} d \tilde{I}_{1} / d t$, which is of the first order in $\gamma$ ).

Equation (B1) can be easily solved if one writes $\tilde{\theta}_{1}=$ $\kappa(\tilde{T}) \tilde{T}_{1}$ in the form of a series expansion as

$$
\tilde{\theta}_{1}=\sum_{\nu} \tilde{A}_{\nu}(t) \tilde{\psi}_{\nu}(\eta)
$$

Inserting the solution of Eq. (B1) found in this way into Eq. (B2) one may rewrite this equation to read

$$
\mathcal{L} \frac{d \tilde{I}_{1}}{d t}+R\{\tilde{T}\} \tilde{I}_{1}+\frac{2 \bar{I}}{l_{0}^{2}} \sum_{\nu} \frac{\tilde{\rho}_{\nu} \tilde{R}_{\nu}}{\tilde{\lambda}_{\nu}} \tilde{I}_{1}=-\sum_{\nu} \frac{\tilde{R}_{\nu} \tilde{F}_{\nu}}{\tilde{\lambda}_{\nu}} .
$$

Here $\tilde{\psi}_{\nu}$ and $\tilde{\lambda}_{\nu}$ are eigenfunctions and eigenvalues of the operator $\hat{H}_{\beta}$ (see Eq. A2 ) in which $\bar{T}$ and $\bar{I}(V)$ are changed to $\tilde{T}$ and $\tilde{I}(U)$, while

$$
\tilde{F}_{\nu}=\left\langle F(t, \eta) \tilde{\psi}_{\nu}\right\rangle ; \tilde{R}_{\nu}=\left\langle\frac{\rho^{\prime}(\tilde{T})}{\kappa(\tilde{T})} \tilde{\psi}_{\nu}\right\rangle ; \tilde{\rho}=\left\langle\rho(\tilde{T}) \tilde{\psi}_{\nu}\right\rangle
$$

Using now Eq. (A7) (in which all quantities marked with the bar sign should be changed to those marked with the tilde sign) one finds the equation for $\tilde{I}_{1}$ to be

$$
\mathcal{L} \frac{d \tilde{I}_{1}}{d t}+\frac{d \tilde{U}}{d \tilde{I}} \tilde{I}_{1}=-\sum_{\nu} \frac{\tilde{R}_{\nu} F_{\nu}}{\tilde{\lambda}_{\nu}}
$$

Solving the linear differential equation (B7) one finds the solution of the linear differential equations (B1) and (B2) for the current $\tilde{I}_{1}(t)$ to be

$$
\begin{gathered}
\tilde{I}_{1}(t)=\exp \{\Gamma(t)\} \\
\times\left\{-\frac{1}{\mathcal{L}} \int_{0}^{t} d \tau \sum_{\nu} \frac{\tilde{R}_{\nu} \tilde{F}_{\nu}}{\tilde{\lambda}_{\nu}} \exp \{-\Gamma(\tau)\}+\tilde{I}_{1}(0)\right\}
\end{gathered}
$$

where

$$
\Gamma(t)=-\frac{1}{\mathcal{L}} \int_{0}^{t} \frac{d \tilde{U}}{d \tilde{I}} d \tau^{\prime}
$$

and $\tilde{I}_{1}(0)$ is the initial value of $\tilde{I}_{1}(t)$. From Eqs. (B88) and (B9) it follows that the current $\tilde{I}_{1}(t)$ grows exponentially with time if the differential resistance is negative (i.e. if $d \tilde{U} / d \tilde{I}<0)$. In order to prevent this increase we impose a condition on the parameters of the zero approximation incorporated in $\tilde{F}_{\nu}$. The condition is expressed by the equation

$$
-\frac{1}{\mathcal{L}} \int_{0}^{\infty} d \tau \sum_{\nu} \frac{\tilde{R}_{\nu} \tilde{F}_{\nu}}{\tilde{\lambda}_{\nu}} e^{-\Gamma(\tau)}+\tilde{I}_{1}(0)=0
$$

As a result one gets

$$
\tilde{I}_{1}=\frac{1}{\mathcal{L}} e^{\Gamma(t)} \int_{t}^{\infty} d \tau \sum_{\nu} \frac{\tilde{R}_{\nu} \tilde{F}_{\nu}}{\tilde{\lambda}_{\nu}} e^{-\Gamma(\tau)}
$$

It follows from Eq. (B10) and Eq. (B11) that the inequality $\left|\tilde{I}_{1}\right| \ll|\tilde{I}|$ is satisfied at all times if

$$
\sum_{\nu} \frac{\tilde{R}_{\nu} \tilde{F}_{\nu}}{\tilde{\lambda}_{\nu}} \sim\left|\tilde{I}_{1}\right| /|\tilde{I}| \ll 1
$$

Therefore, the parameters of the zero approximation $\tilde{I}(t)$ and $\tilde{U}(t)$ should satisfy the equation

$$
\sum_{\nu} \frac{\tilde{R}_{\nu} \tilde{F}_{\nu}}{\tilde{\lambda}_{\nu}}=0
$$

Using Eq. (B12) and Eqs. (10), (A7), (B3), and (B6) we find the final set of equations Eq.(32) for the current $\tilde{I}(t)$ and the voltage $U(t)$. 
1 M. N. Baibich, J. M. Broto, A. Fert, F. Nguyen Van Dau, and F. Petroff, Phys. Rev. Lett. 61, 2472 (1988).

2 J. Barnas, A. Fuss, R. Camley, P. Grunberg, and W. Zinn, Phys. Rev. B 42, 8110 (1990).

3 J. C. Slonczewski, J. Magn. Magn. Mater. 159, L1 (1996); ibid. 195, L261 (1999).

${ }^{4}$ L. Berger, Phys. Rev. B 54, 9353 (1996).

5 M. Tsoi, A.G.M. Jansen, J. Bass, W.-C. Chiang, M. Seck, V. Tso, and P.Wyder, Phys. Rev. Lett. 80, 4281 (1998).

6 M. Tsoi, A.G.M. Jansen, J. Bass, W.-C. Chiang, V. Tsoi, and P.Wyder, Nature (London), 406, 46 (2000).

7 E.B. Myers, D.C. Ralph,J.A. Katine, R.N. Louie, and R.A. Buhrman Science 285, 867 (1999).

8 J.A. Katine, F.J. Albert, R.A. Buhrman, E.B. Myers, and D.C. Ralph, Phys. Rev. Lett. 84, 3149 (2000).

9 S.I. Kiselev, J.C. Sankey, I.N. Krivorotov, N.C. Emley, R.J. Schoelkopf, R.A. Buhrman, and D.C. ralph, Nature (London) 425, 380 (2003).

10 W.H. Rippard, M.R. Pufall, and T.J. Silva, Appl. Phys. Lett. 82, 1260 (2003).

11 I. K. Yanson, Y. G. Naidyuk, D. L. Bashlakov, V.V. Fisun, O.P. Balkashin, V. Korenivski, A. Konovalenko, and R.I. Shekhter, Phys. Rev. Lett. 95, 186602 (2005).

12 I.O. Kulik, Phys. Lett. 106A, 187 (1984).

13 I.F. Itskovitch, M.V. Moskalets, R.I. Shekhter, and I.O. Kulik Sov. J. Low Temp. Phys.13, 588 (1987).

14 S.I. Kulinich, R.I. Shekhter, and I.V. Krive, Low Temp. Physics 26, 437 (2000).

15 A. Vl. Gurevich and R. G. Mints, Rev. Mod. 59, 941 (941).

16 Yu. G. Naidiuk, K. Gloos, I. K. Yanson, and N. K. Sato,
J. Phys.: Condensed Matter 163433 (2004).

17 A. A. Slutskin and A. M. Kadigrobov, JETP Lett. 28,201 (1978).

18 A. M. Kadigrobov, A. A. Slutskin, and I. V. Krivoshei, Sov. Phys. JETP 60, 754 (1984).

19 V. V. Boiko, Yu. F. Podrezov, and N. P. Klimova, Sov. Phys. JETP 35, 649 (1982).

$20 \mathrm{Yu}$. N. Chiang and I. I. Logvinov, Sov. J. Low Temp. Phys. 8, 388 (1982).

21 G. I. Abramov, A. Vl. Gurevich, V. M. Dzigutov, R. G. Mints, and L. M. Fisher, JETP Lett. 37, 535 (1983).

22 A. M. Kadigrobov, Yu. N. Chiang, and I. I. Logvinov, Sov. Phys. Solid State 28, 1903 (1986).

23 V. N. Morgun, V. A. Bondar, A. M. Kadigrobov, and N. N. Chebotaev, Sov. Phys. Solid State 35, 31 (1993).

${ }^{24}$ L. D. Landau and E. M. Lifshits, Electrodynamics of Continuous Media, Pergamon Press, Oxford (1998).

25 I. O. Kulik, Phys. Lett. A 106, 187 (1984).

26 B. I. Verkin, I. K. Yanson, I. O. Kulik, O. I. Shklyarevskii, A. A. Lysykh, and Yu. G. Naidyuk, Solid State Commun. 30, 215 (1979).

27 I. O. Kulik, Sov. J. Low Temp. Phys. 18, 302 (1992).

28 N. Kohlrausch, Ann. Physik (Leipzig) 1, 132 (1900)

29 F. J. Blatt "Physics of electronic conduction in solids", McGraw-Hill Book Company, 1968.

30 A. A. Andronov, A. A. Witt, and S. E. Khaikin, "Theory of Oscillations" (Pergamon, Oxford) 1966.

31 P. Bruno, Phys. Rev. Letters 83, 2425 (1999). 